\title{
Existence of Solutions for Fractional Differential Inclusions with Separated Boundary Conditions in Banach Space
}

\author{
Mabrouk Bragdi, ${ }^{1}$ Amar Debbouche, ${ }^{2}$ and Dumitru Baleanu ${ }^{3,4,5}$ \\ ${ }^{1}$ Department of Mathematics, Laboratory of Dynamical Systems and Control, Larbi Ben M'hidi University, P.O. Box 358, \\ Oum El Bouaghi, Algeria \\ ${ }^{2}$ Department of Mathematics, Guelma University, 24000 Guelma, Algeria \\ ${ }^{3}$ Department of Mathematics and Computer Sciences, Cankaya University, 06530 Ankara, Turkey \\ ${ }^{4}$ Department of Chemical and Materials Engineering, Faculty of Engineering, King Abdulaziz University, P.O. Box 80204, \\ Jeddah 21589, Saudi Arabia \\ ${ }^{5}$ Institute of Space Sciences, Magurele-Bucharest, Romania
}

Correspondence should be addressed to Amar Debbouche; amar_debbouche@yahoo.fr

Received 10 March 2013; Accepted 11 May 2013

Academic Editor: Changpin Li

Copyright (c) 2013 Mabrouk Bragdi et al. This is an open access article distributed under the Creative Commons Attribution License, which permits unrestricted use, distribution, and reproduction in any medium, provided the original work is properly cited.

We discuss the existence of solutions for a class of some separated boundary differential inclusions of fractional orders $2<\alpha<3$ involving the Caputo derivative. In order to obtain necessary conditions for the existence result, we apply the fixed point technique, fractional calculus, and multivalued analysis.

\section{Introduction}

In recent years a great interest was devoted to the study of (singular and Neumann) boundary-value problems of fractional order [1-8], see also [9-12]. In the literature of fractional calculus, there are several definitions of fractional derivative that can be used. However, the most popular senses are the Riemann-Liouville and Caputo fractional derivatives; see, for instance, [13-19].

In this paper, we use the Caputo's fractional derivative since mathematical modeling of many physical problems requires initial and boundary conditions. These demands are satisfied using the Caputo fractional derivative. For more details we refer the reader to $[20,21]$ and references therein.

The importance of fractional boundary-value problems stems from the fact that they model various applications in fluid mechanics, viscoelasticity, physics, biology, and economics which cannot be modeled by differential equations with integer derivatives [21-23].

Delbosco and Rodino [24] considered the existence of a solution for the nonlinear fractional differential equation $d^{\alpha} x(t) / d t^{\alpha}=f(t, x)$, where $0<\alpha<1$ and $f:[0, a] \times \mathbb{R} \rightarrow$
$\mathbb{R}, 0<a \leq+\infty$, is a given function, continuous in $(0, a) \times \mathbb{R}$. They obtained results for solutions by using the Schauder fixed point theorem and the Banach contraction principle. Qiu and Bai [25] considered the existence of positive solution for equation:

$$
\begin{gathered}
\frac{d^{\alpha} x(t)}{d t^{\alpha}}+f(t, x(t))=0, \quad 0<t<1, \\
x(0)=x^{\prime}(1)=x^{\prime \prime}(0)=0,
\end{gathered}
$$

where $2<\alpha \leq 3$ and $f:(0,1] \times[0, \infty) \rightarrow[0, \infty)$ with $f$ being singular at $t=0$ (i.e., $\lim _{t \rightarrow 0^{+}} f(t, \cdot)=+\infty$ ), by using Krasnoselskii's fixed point theorem and nonlinear alternative of Leray-Schauder type in a cone. Recently, Aitaliobrahim [26] considered the existence of solutions to the boundaryvalue problem:

$$
\begin{gathered}
x^{\prime \prime}(t) \in F\left(t, x(t), x^{\prime}(t)\right), \quad \text { a.e. on }[0,1], \\
x^{\prime}(0)=r, \quad x^{\prime}(1)=s,
\end{gathered}
$$


where $F$ is a closed multifunction, measurable in the first argument, and Lipschitz continuous in the second argument, by using fixed point theory for multivalued maps.

Motivated by the previous results, in this work we establish the existence result of a new version of fractional separated boundary-value problem:

$$
\begin{gathered}
\frac{d^{\alpha} x(t)}{d t^{\alpha}} \in F\left(t, x(t), x^{\prime}(t), x^{\prime \prime}(t)\right), \quad \text { a.e. on }[0,1], \\
x(0)=0, \quad x^{\prime}(1)=s, \quad x^{\prime \prime}(0)=r,
\end{gathered}
$$

where $2<\alpha \leq 3, F$ is nonconvex, closed multifunction, measurable in the first argument, and Lipschitz continuous in the second argument, and $r, s$ are in a Banach space $X$. The work is organized as follows. In Section 2, we recall some preliminary facts that we need in the sequel while in Section 3, we give the main result. Finally in Section 4 we give example to illustrate the application of our results.

\section{Preliminaries}

In this section, we present basic definitions of fractional calculus and some essential facts from multivalued analysis that will be used in this work to obtain our main results.

Definition 1. A real function $f(t), t>0$, is said to be in the space $C_{\mu}, \mu \in \mathbb{R}$, if there exists a real number $p>\mu$, such that $f(t)=t^{p} f_{1}(t)$, where $f_{1}(t) \in C[0, \infty)$, and it is said to be in the space $C_{\mu}^{m}$ if $f^{(m)} \in C_{\mu}, m \in \mathbb{N}$.

Definition 2. The left Riemann-Liouville fractional integral of order $\alpha>0$, of a function $f \in C_{\mu}, \mu \geq-1$, is defined by

$$
I^{\alpha} f(t)=\frac{1}{\Gamma(\alpha)} \int_{0}^{t}(t-s)^{\alpha-1} f(s) d s, \quad t>0 .
$$

Definition 3. For $\alpha>0, m-1<\alpha<m, m \in \mathbb{N}, t>0$, and $f \in C_{-1}^{m}$, the left Caputo fractional derivative is defined by

$$
D^{\alpha} f(t)=\frac{1}{\Gamma(m-\alpha)} \int_{0}^{t}(t-s)^{m-1-\alpha} f^{(m)}(s) d s,
$$

where $\Gamma$ is the well-known Gamma function.

The Caputo derivative defined in (5) is related to the Riemann-Liouville fractional integral, $I^{\alpha}$, of order $\alpha \in \mathbb{R}^{+}$, by

$$
D^{\alpha} f(t)=I^{m-\alpha} f^{(m)}(t) .
$$

It is known (see [20]) that

$$
\begin{gathered}
I^{\alpha}\left(D^{\alpha} f(t)\right)=f(t)-\sum_{k=0}^{m-1} c_{k} t^{k}, \\
D^{\alpha} I^{\alpha} f(t)=f(t),
\end{gathered}
$$

where in (7), $c_{k}=f^{(k)}(0+) / k !, 0 \leq k \leq m-1$.
Now, let $X$ be a real separable Banach space with the norm $\|\cdot\|$. We denote by $\mathscr{C}([0,1], X)$ the Banach space of continuous functions from $[0,1]$ to $E$ equipped with the norm $\|x(\cdot)\|_{\infty}:=\sup \{\|x(t)\| ; t \in[0,1]\}$. For $x \in X$ and for nonempty sets $A, B$ of $X$ we denote $d(x, A)=$ $\inf \{d(x, y) ; y \in A\}, e(A, B):=\sup \{d(x, B) ; x \in A\}$, and $H(A, B):=\max \{e(A, B), e(B, A)\}$. A multifunction is said to be measurable if its graph is measurable.

Also, we recall the following results that will be used in this paper.

Definition 4 (see [26]). Let $T: X \rightarrow 2^{X}$ be a multifunction with closed values:

(i) $T$ is $k$-Lipschitz if $H(T(x), T(y)) \leq k\|x-y\|$ for each $x, y \in X$,

(ii) $T$ is a contraction if it is $k$-Lipschitz with $k<1$,

(iii) $T$ has a fixed point if there exists $x \in X$ such that $x \in$ $T(x)$.

Lemma 5 (see [26]). If $T: X \rightarrow 2^{X}$ is a contraction with nonempty closed values, then it has a fixed point.

Lemma 6 (see [26]). Assume that $F:[a, b] \times X \rightarrow 2^{X}$ is a multifunction with nonempty closed values satisfying the following:

(i) for every $x \in X, F(\cdot, x)$ is measurable on $[a, b]$;

(ii) for everyt $\in[a, b], F(t, \cdot)$ is (Hausdorff) continuous on E.

Then for any measurable function $x(\cdot):[a, b] \rightarrow X$, the multifunction $F(\cdot, x(\cdot))$ is measurable on $[a, b]$.

Definition 7 (see [26]). A measurable multivalued function $F:[0,1] \rightarrow 2^{X}$ is said to be integrably bounded if there exists a function $h \in L^{1}([0,1], X)$ such that for all $v \in F(t),\|v\| \leq$ $h(t)$ for almost every $t \in[0,1]$.

Definition 8 (see [26]). A function $x(\cdot):[0,1] \rightarrow X$ is said to be a solution of (3) if $x(\cdot)$ is absolutely continuous on $[0,1]$ and satisfies (3).

Lemma 9 (see [25]). Given $\varphi \in C[0,1]$ and $2<\alpha \leq 3$, the unique solution of

$$
\begin{gathered}
\frac{d^{\alpha} y(t)}{d t^{\alpha}}+\varphi(t)=0, \quad 0<t<1, \\
y(0)=y^{\prime}(1)=y^{\prime \prime}(0)=0
\end{gathered}
$$

is

$$
y(t)=\int_{0}^{1} G(t, s) \varphi(s) d s
$$


where

$$
\begin{aligned}
& G(t, s) \\
& \quad= \begin{cases}\frac{(\alpha-1) t(1-s)^{\alpha-2}-(t-s)^{\alpha-1}}{\Gamma(\alpha)}, & 0 \leq s \leq t \leq 1, \\
\frac{t(1-s)^{\alpha-2}}{\Gamma(\alpha-1)}, & 0 \leq t \leq s \leq 1 .\end{cases}
\end{aligned}
$$

Obviously, $G(t, s)$ is continuous on $[0,1] \times[0,1]$ and $0<$ $G(t, s) \leq \lambda$, for each $t, s \in[0,1]$ and some $\lambda$.

\section{Main Results}

Now we are in a position to state and prove the main results of this paper.

Theorem 10. Let $F:[0,1] \times X \times X \times X \rightarrow 2^{X}$ be a set-valued map with nonempty closed values satisfying the following:

(i) for each $(x, y, z) \in X \times X \times X, t \mapsto F(t, x, y, z)$ is measurable and integrably bounded;

(ii) there exists a function $m(\cdot) \in L^{1}\left([0,1], \mathbb{R}^{+}\right)$such that for all $t \in[0,1]$ and for all $x_{1}, x_{2}, y, z \in X$

$$
H\left(F\left(t, x_{1}, y, z\right), F\left(t, x_{2}, y, z\right)\right) \leq m(t)\left\|x_{1}-x_{2}\right\| \text {. }
$$

Then, if $\int_{0}^{1}(1+m(s)) d s<1 / \lambda$, for all $r, s \in X$, the problem (3) has at least one solution on $[0,1]$.

Proof. For the proof of this theorem, we use the similar steps as those of [26, Theorem 2.6] together with the theory of fractional calculus. Let $r, s$ be in $X$. We introduce first the function $\rho:[0,1] \rightarrow X$ defined by

$$
\rho(t)=(r+s) t-\frac{1}{2} r t^{2}, \quad \forall t \in[0,1],
$$

and the multifunction $H:[0,1] \times \mathscr{C}([0,1], X) \rightarrow 2^{X}$ defined by

$$
\begin{aligned}
& H(t, y(\cdot)) \\
& =\varphi(t)-F\left(t, y(t)+\rho(t), y^{\prime}(t)\right. \\
& \left.+\rho^{\prime}(t), y^{\prime \prime}(t)+\rho^{\prime \prime}(t)\right),
\end{aligned}
$$

for all $(t, y(\cdot)) \in[0,1] \times \mathscr{C}([0,1], X)$. Consider the following problem:

$$
\begin{gathered}
-\frac{d^{\alpha} y(t)}{d t^{\alpha}}+\varphi(t) \in H(t, y(\cdot)), \quad \text { a.e. on }[0,1], \\
y(0)=y^{\prime}(1)=y^{\prime \prime}(0)=0 .
\end{gathered}
$$

We should note that the function $y(\cdot)$ is a solution of (15), if and only if the function $x(t)=y(t)+\rho(t)$ is a solution of (3), for all $t \in[0,1]$.

Next, by Lemma 6 , for $y(\cdot) \in \mathscr{C}([0,1], X), F\left(\cdot, y(\cdot), y^{\prime}(\cdot)\right.$, $\left.y^{\prime \prime}(\cdot)\right)$ is closed and measurable; then it has a measurable selection which, by hypothesis (i), belongs to $L^{1}([0,1], X)$. Thus the set

$S_{F, y(\cdot)}$

$$
\begin{aligned}
:=\left\{f \in L^{1}([0,1], X):\right. & \\
& \left.\quad f(t) \in F\left(t, y(t), y^{\prime}(t), y^{\prime \prime}(t)\right) \text { for a.e. } t \in[0,1]\right\}
\end{aligned}
$$

is nonempty. Let us transform problem (15) into a fixed point problem. Consider the multivalued map

$$
T: \mathscr{C}([0,1], X) \longrightarrow 2^{\mathscr{C}([0,1], X)}
$$

defined as follows, for $y(\cdot) \in \mathscr{C}([0,1], X)$ :

$T(y(\cdot))$

$$
\begin{aligned}
=\{z(\cdot) \in \mathscr{C}([0,1], X): \\
\left.\quad z(t)=\int_{0}^{1} G(t, s) h(s) d s, \forall t \in[0,1], h \in S_{H, y(\cdot)}\right\},
\end{aligned}
$$

where

$$
\begin{aligned}
S_{H, y(\cdot)}:=\left\{h \in L^{1}([0,1], X):\right. \\
\quad h(t) \in H(t, y(\cdot)) \text { for a.e. } t \in[0,1]\} .
\end{aligned}
$$

We will show that $T$ satisfies the assumptions of Lemma 5. The proof will be given in two steps.

Step 1 (Thas nonempty closed values). Indeed, let $\left(y_{p}(\cdot)\right){ }_{p \geq 0} \epsilon$ $T(y(\cdot))$ such that $\left(y_{p}(\cdot)\right)_{p \geq 0}$ converges to $\bar{y}(\cdot)$ in $\mathscr{C}([0,1], X)$. Then $\bar{y}(\cdot) \in \mathscr{C}([0,1], X)$ and for each $t \in[0,1]$,

$$
y_{p}(t) \in \int_{0}^{1} G(t, s) H(s, y(\cdot)) d s,
$$

where $\int_{0}^{1} G(t, s) H(s, y(\cdot)) d s$ is the Aumann integral of $G(t, \cdot)$ $H(\cdot, y)$, which is defined as

$$
\begin{aligned}
\int_{0}^{1} G & (t, s) H(s, y(\cdot)) d s \\
& =\left\{\int_{0}^{1} G(t, s) h(s) d s, h \in S_{H, y(\cdot)}\right\} .
\end{aligned}
$$

Using the fact that the set-valued map $F$ is closed and by (14), we conclude that the set

$$
\int_{0}^{1} G(t, s) H(s, y(\cdot)) d s
$$

is closed for all $t \in[0,1]$. Then

$$
\bar{y}(t) \in \int_{0}^{1} G(t, s) H(s, y(\cdot)) d s .
$$


So, there exists $h \in S_{H, y(\cdot)}$ such that

$$
\bar{y}(t)=\int_{0}^{1} G(t, s) h(s) d s
$$

Hence $\bar{y}(\cdot) \in T(y(\cdot))$. So $T(y(\cdot))$ is closed for each $y(\cdot) \in$ $\mathscr{C}([0,1], X)$.

Step 2 ( $T$ is a contraction). Indeed, let $y_{1}(\cdot), y_{2}(\cdot) \in$ $\mathscr{C}([0,1], X)$ and consider $z_{1}(\cdot) \in T\left(y_{1}(\cdot)\right)$. Then there exists $h_{1} \in S_{H, y_{1}(\cdot)}$ such that

$$
z_{1}(t)=\int_{0}^{1} G(t, s) h_{1}(s) d s, \quad \forall t \in[0,1] .
$$

Using (14), there exists $f_{1} \in S_{F, y_{1}(\cdot)}$ such that

$$
h_{1}(t)=y_{1}(t)-f_{1}(t), \quad \forall t \in[0,1] .
$$

On the other hand, let $\varepsilon>0$ and consider the valued map $U_{\varepsilon}:[0,1] \rightarrow 2^{X}$, given by

$$
U_{\varepsilon}(t)=\left\{x \in X:\left\|f_{1}(t)-x\right\| \leq m(t)\left\|y_{1}(t)-y_{2}(t)\right\|+\varepsilon\right\} .
$$

We claim that $U_{\varepsilon}(t)$ is nonempty, for each $t \in[0,1]$. Indeed, let $t \in[0,1]$; then we have

$$
\begin{aligned}
& H\left(F\left(t, y_{1}(t), y_{1}^{\prime}(t), y_{1}^{\prime \prime}(t)\right), F\left(t, y_{2}(t), y_{2}^{\prime}(t), y_{2}^{\prime \prime}(t)\right)\right) \\
& \quad \leq m(t)\left\|y_{1}(t)-y_{2}(t)\right\| .
\end{aligned}
$$

Hence, there exists $x \in F\left(t, y_{2}(t), y_{2}^{\prime}(t), y_{2}^{\prime \prime}(t)\right)$, such that

$$
\left\|f_{1}(t)-x\right\| \leq m(t)\left\|y_{1}(t)-y_{2}(t)\right\|+\varepsilon
$$

The multifunction

$$
V: t \longrightarrow U_{\varepsilon}(t) \cap F\left(t, y_{2}(t), y_{2}^{\prime}(t), y_{2}^{\prime \prime}(t)\right) \text { is measurable. }
$$

Then there exists a measurable selection for $V$ denoted by $f_{2}$ such that, for all $t \in[0,1]$,

$$
f_{2}(t) \in F\left(t, y_{2}(t), y_{2}^{\prime}(t), y_{2}^{\prime \prime}(t)\right)
$$

and

$$
\left\|f_{1}(t)-f_{2}(t)\right\| \leq m(t)\left\|y_{1}(t)-y_{2}(t)\right\|+\varepsilon .
$$

Now, for all $t \in[0,1]$, set $h_{2}(t)=y_{2}(t)-f_{2}(t)$ and

$$
z_{2}(t)=\int_{0}^{1} G(t, s) h_{2}(s) d s
$$

We have

$$
\begin{aligned}
\| z_{1}(t) & -z_{2}(t) \| \\
\leq & \int_{0}^{1}\|G(t, s)\|\left\|h_{1}(s)-h_{2}(s)\right\| d s \\
\leq & \lambda \int_{0}^{1}\left\|y_{1}(s)-y_{2}(s)\right\| d s+\lambda \int_{0}^{1}\left\|f_{1}(s)-f_{2}(s)\right\| d s \\
\leq & \lambda \int_{0}^{1}\left\|y_{1}(s)-y_{2}(s)\right\| d s \\
& +\lambda \int_{0}^{1} m(s)\left\|y_{1}(s)-y_{2}(s)\right\| d s+\lambda \varepsilon \\
\leq & \lambda\left\|y_{1}(\cdot)-y_{2}(\cdot)\right\|_{\infty} \int_{0}^{1}(1+m(s)) d s+\lambda \varepsilon .
\end{aligned}
$$

So, we conclude that

$$
\left\|z_{1}(\cdot)-z_{2}(\cdot)\right\|_{\infty} \leq \lambda\left\|y_{1}(\cdot)-y_{2}(\cdot)\right\|_{\infty} \int_{0}^{1}(1+m(s)) d s+\lambda \varepsilon .
$$

By an analogous relation, obtained by interchanging the roles of $y_{1}(\cdot)$ and $y_{2}(\cdot)$, it follows that

$$
\begin{aligned}
& H\left(T\left(y_{1}(\cdot)\right), T\left(y_{2}(\cdot)\right)\right) \\
& \quad \leq \lambda\left\|y_{1}(\cdot)-y_{2}(\cdot)\right\|_{\infty} \int_{0}^{1}(1+m(s)) d s+\lambda \varepsilon .
\end{aligned}
$$

By letting $\varepsilon \rightarrow 0$, we obtain

$$
\begin{aligned}
& H\left(T\left(y_{1}(\cdot)\right), T\left(y_{2}(\cdot)\right)\right) \\
& \quad \leq \lambda\left\|y_{1}(\cdot)-y_{2}(\cdot)\right\|_{\infty} \int_{0}^{1}(1+m(s)) d s .
\end{aligned}
$$

Consequently, if $\int_{0}^{1}(1+m(s)) d s<1 / \lambda, T$ is a contraction. By Lemma 5, $T$ has a fixed point which is a solution of (15).

\section{Example}

In this section we present an example to illustrate the applications of our main results, and we consider the following fractional inclusion boundary-value problem:

$$
\frac{d^{\alpha} x(t)}{d t^{\alpha}} \in F\left(t, x(t), x^{\prime}(t), x^{\prime \prime}(t)\right), \quad t \in[0,1], 2<\alpha \leq 3,
$$

$$
x(0)=0, \quad x^{\prime}(1)=s, \quad x^{\prime \prime}(0)=r,
$$

where $r$ and $s$ are arbitrary real numbers and $F:[0,1] \times \mathbb{R} \times$ $\mathbb{R} \times \mathbb{R} \rightarrow \mathscr{P}(\mathbb{R})$ is a multivalued map given by

$$
F(t, x, y, z)=\left[0, \frac{\sin x}{(1+t)^{2}}+\cos (y z)\right] \text {. }
$$


Then we have

$$
\begin{gathered}
\sup \{|u|: u \in F(t, x, y, z)\} \leq 1+\frac{1}{(1+t)^{2}}, \\
H(F(t, x, y, z), F(t, \bar{x}, y, z)) \leq \frac{1}{(1+t)^{2}}|x-\bar{x}| .
\end{gathered}
$$

Let $m(t)=1 /(1+t)^{2}$. Then $\int_{0}^{1}(1+m(s)) d s=3 / 2<1 / \lambda$ for some $\lambda>2 / 3$.

By Theorem 10 the problem (38) has at least one solution on $[0,1]$.

\section{References}

[1] R. P. Agarwal, D. O’Regan, and S. Staněk, "Positive solutions for mixed problems of singular fractional differential equations," Mathematische Nachrichten, vol. 285, no. 1, pp. 27-41, 2012.

[2] B. Ahmad, J. J. Nieto, and J. Pimentel, "Some boundary value problems of fractional differential equations and inclusions," Computers \& Mathematics with Applications, vol. 62, no. 3, pp. 1238-1250, 2011.

[3] B. Ahmad and S. K. Ntouyas, "A note on fractional differential equations with fractional separated boundary conditions," Abstract and Applied Analysis, vol. 2012, Article ID 818703, 11 pages, 2012.

[4] Z. Bai and W. Sun, "Existence and multiplicity of positive solutions for singular fractional boundary value problems," Computers \& Mathematics with Applications, vol. 63, no. 9, pp. 1369-1381, 2012.

[5] J. Caballero, J. Harjani, and K. Sadarangani, "Positive solutions for a class of singular fractional boundary value problems," Computers \& Mathematics with Applications, vol. 62, no. 3, pp. 1325-1332, 2011.

[6] I. J. Cabrera, J. Harjani, and K. B. Sadarangani, "Existence and uniqueness of positive solutions for a singular fractional threepoint boundary value problem," Abstract and Applied Analysis, vol. 2012, Article ID 803417, 18 pages, 2012.

[7] J. Jin, X. Liu, and M. Jia, "Existence of positive solutions for singular fractional differential equations with integral boundary conditions," Electronic Journal of Differential Equations, vol. 2012, no. 63, pp. 1-14, 2012.

[8] D. O'Regan and S. Stanek, "Fractional boundary value problems with singularities in space variables," Nonlinear Dynamics, vol. 71, no. 4, pp. 641-652, 2013.

[9] B. Ahmad and S. K. Ntouyas, "Boundary value problems for $n$-th order differential inclusions with four-point integral boundary conditions," Opuscula Mathematica, vol. 32, no. 2, pp. 205-226, 2012.

[10] A. Boucherif and N. Al-Malki, "Solvability of Neumann boundary-value problems with Carathéodory nonlinearities," Electronic Journal of Differential Equations, vol. 2004, no. 51, pp. $1-7,2004$

[11] G. A. Chechkin, D. Cioranescu, A. Damlamian, and A. L. Piatnitski, "On boundary value problem with singular inhomogeneity concentrated on the boundary," Journal de Mathématiques Pures et Appliquées, vol. 98, no. 2, pp. 115-138, 2012.

[12] A. Nouy, M. Chevreuil, and E. Safatly, "Fictitious domain method and separated representations for the solution of boundary value problems on uncertain parameterized domains," Computer Methods in Applied Mechanics and Engineering, vol. 200, no. 45-46, pp. 3066-3082, 2011.
[13] M. Bragdi and M. Hazi, "Existence and uniqueness of solutions of fractional quasilinear mixed integrodifferential equations with nonlocal condition in Banach spaces," Electronic Journal of Qualitative Theory of Differential Equations, vol. 2012, no. 51, pp. 1-16, 2012.

[14] A. Debbouche and D. Baleanu, "Controllability of fractional evolution nonlocal impulsive quasilinear delay integrodifferential systems," Computers \& Mathematics with Applications, vol. 62, no. 3, pp. 1442-1450, 2011.

[15] A. Debbouche and D. Baleanu, "Exact null controllability for fractional nonlocal integrodifferential equations via implicit evolution system," Journal of Applied Mathematics, vol. 2012, Article ID 931975, 17 pages, 2012.

[16] A. Debbouche, D. Baleanu, and R. P. Agarwal, "Nonlocal nonlinear integrodifferential equations of fractional orders," Boundary Value Problems, vol. 2012, article 78, pp. 1-10, 2012.

[17] C. Kou, H. Zhou, and C. Li, "Existence and continuation theorems of Riemann-Liouville type fractional differential equations," International Journal of Bifurcation and Chaos, vol. 22, no. 4, Article ID 1250077, 12 pages, 2012.

[18] C. Li and Y. Ma, "Fractional dynamical system and its linearization theorem," Nonlinear Dynamics, vol. 71, no. 4, pp. 621-633, 2013.

[19] C. P. Li and F. R. Zhang, "A survey on the stability of fractional differential equations," The European Physical Journal: Special Topics, vol. 193, no. 1, pp. 27-47, 2011.

[20] A. A. Kilbas, H. M. Srivastava, and J. J. Trujillo, Theory and Applications of Fractional Differential Equations, vol. 204 of North-Holland Mathematics Studies, Elsevier, Amsterdam, The Netherlands, 2006.

[21] I. Podlubny, Fractional Differential Equations, Mathematics in Science and Engineering, Academic Press, San Diego, Calif, USA, 1993.

[22] T. M. Atanackovic and B. Stankovic, "Generalized wave equation in nonlocal elasticity," Acta Mechanica, vol. 208, no. 1-2, pp. $1-10,2009$.

[23] M. Caputo, "Linear models of dissipation whose $q$ is almost frequency independent-part II," Geophysical Journal of the Royal Astronomical Society, vol. 13, pp. 529-539, 1967.

[24] D. Delbosco and L. Rodino, "Existence and uniqueness for a nonlinear fractional differential equation," Journal of Mathematical Analysis and Applications, vol. 204, no. 2, pp. 609-625, 1996.

[25] T. Qiu and Z. Bai, "Existence of positive solutions for singular fractional differential equations," Electronic Journal of Differential Equations, vol. 2008, no. 146, pp. 1-9, 2008.

[26] M. Aitaliobrahim, "Neumann boundary-value problems for differential inclusions in banach spaces," Electronic Journal of Differential Equations, vol. 2010, no. 104, pp. 1-5, 2010. 


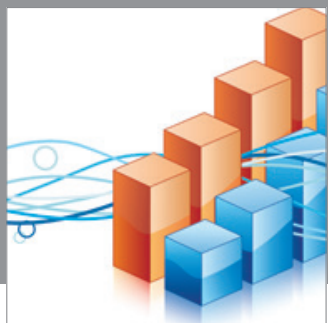

Advances in

Operations Research

mansans

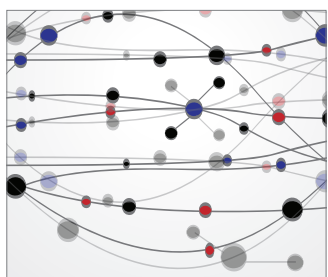

The Scientific World Journal
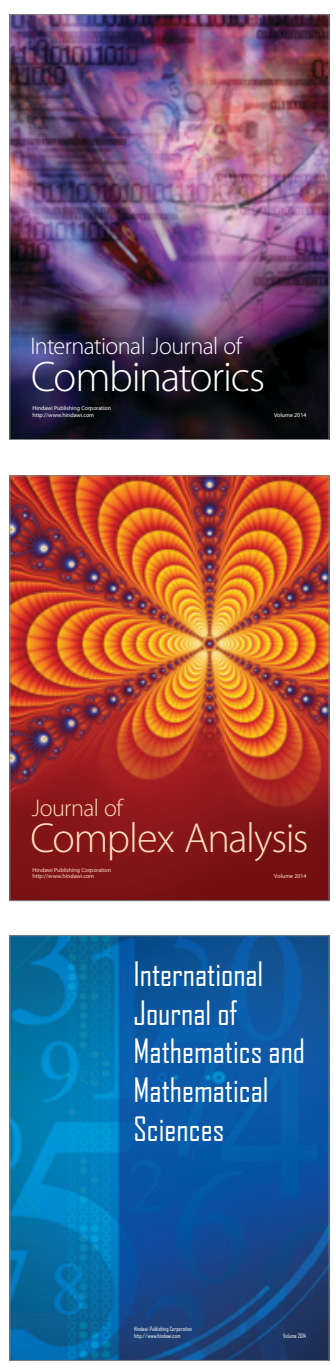
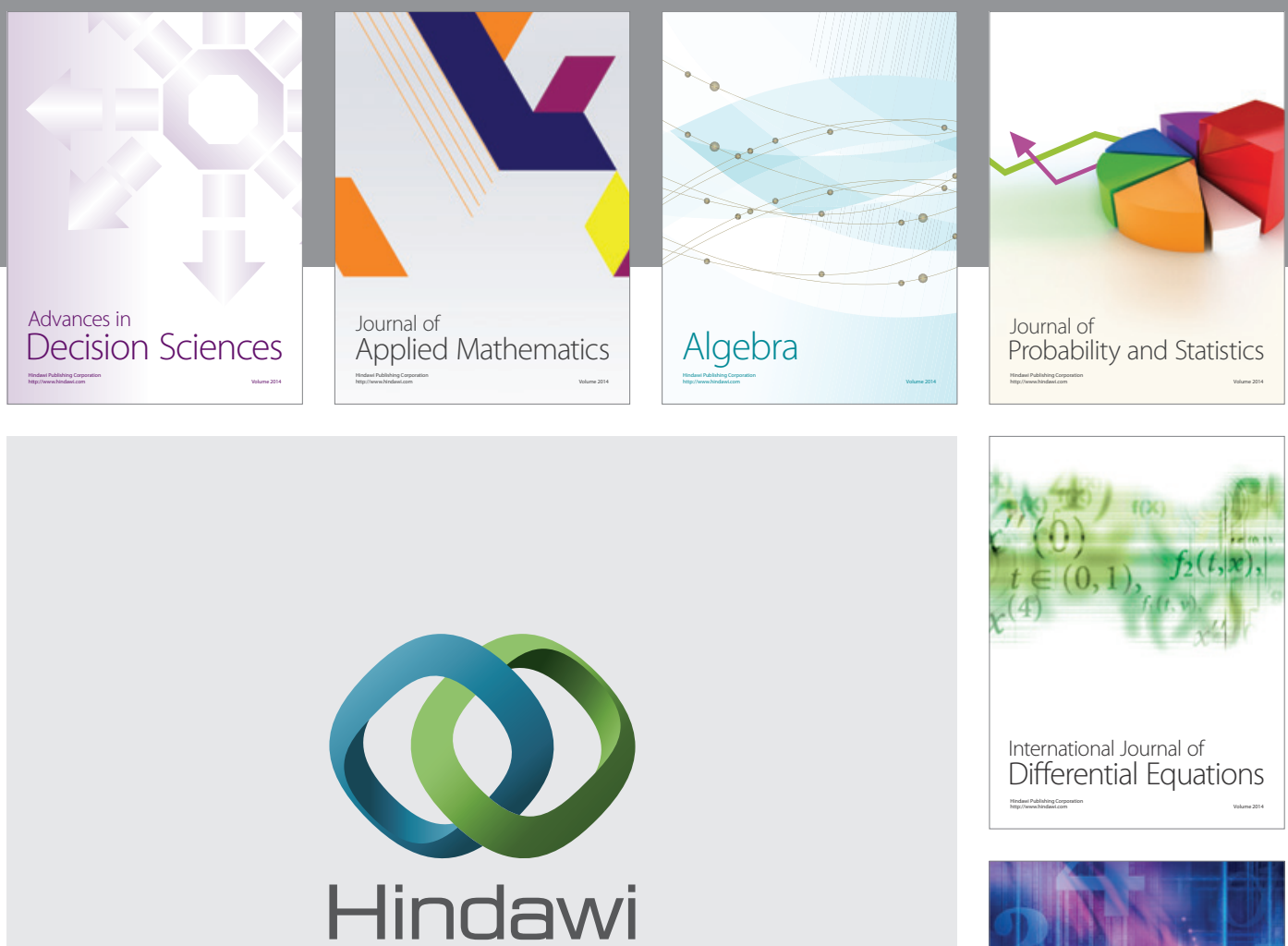

Submit your manuscripts at http://www.hindawi.com
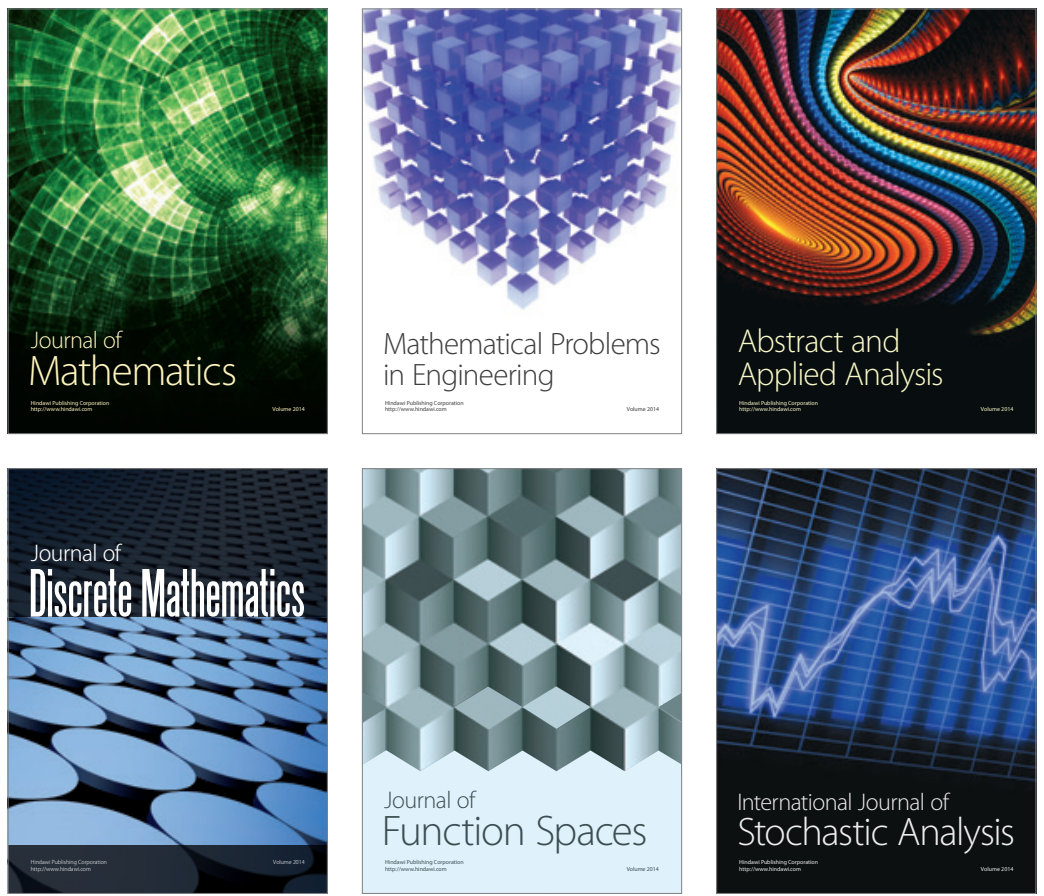

Journal of

Function Spaces

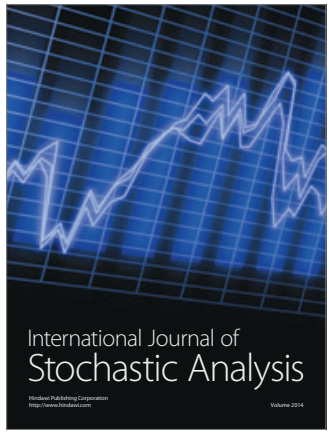

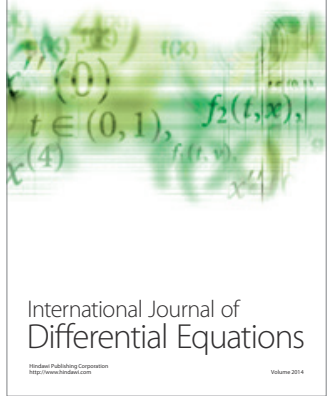
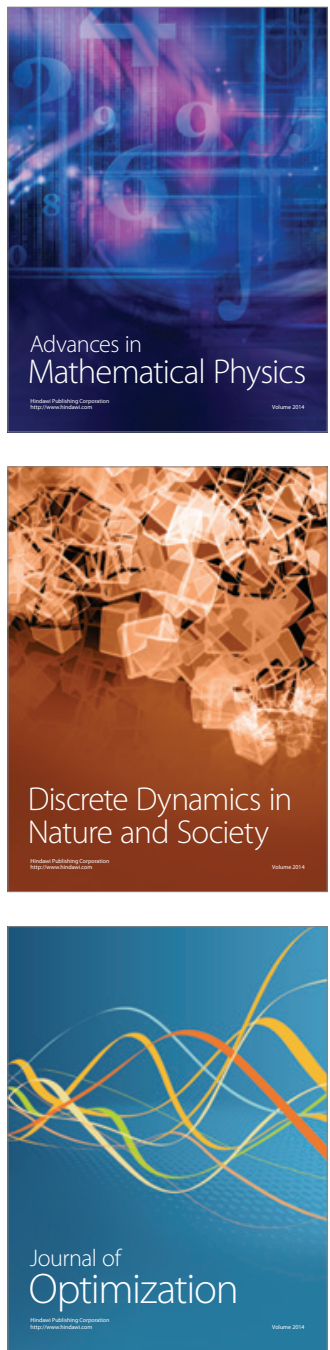